\title{
Table of Contents and
}

\section{Guide to Abstract Numbers}

A message from the Journal of Vestibular Research 49

Table of Contents and Guide to Abstract Numbers 51

Abstracts: Oral Sessions $\quad 53$

$\begin{array}{ll}\text { Abstracts: Poster Sessions } & 141\end{array}$

$\begin{array}{lr}\text { Author Index } & 253\end{array}$

\section{Guide to Abstract Numbers}

Abstracts for oral presentations are designated by a capital letter that corresponds to the topic of the presentation followed by a number. Abstracts for poster presentations are designated by the letter "P" followed by a letter that corresponds to the topic of the presentation followed by a number. The topic designations are as follows:
A. Epidemiology
B. Microgravity Investigations
C. Visual-Vestibular Interaction
D. Vestibular Testing
E. Posturography
F. Utricular and Saccular Testing with VEMP
G. Head Impulse
H. Vestibular Evoked Responses
I. Vestibular Neurophysiology, Morphology and Pathology
J. New Examinations of Vestibular Dysfunction
K. Central and Peripheral Disorders
L. Cochlear-Vestibular Testing
M. Inner Ear Morphology, Imaging
N. Vestibular Loss
O. BPPV
P. Whiplash Injuries, Neck Disorders
Q. Hydrops-Meniere
R. Acoustic Neuromas/Otoneurosurgery
S. Migraine Including Vestibular Migraine
T. Motion Sickness
U. Central Aspects of Vertigo
V. Psychiatric/Physiological Aspects of Vertigo
W. Cognitive/Cortical Aspects of Vertigo
$\mathrm{X}$. Aging and Balance
Y. Intratympanic Treatment
Z. Vestibular Compensation, Rehabilitation and New Treatments 